\title{
How the microbiome is influenced by the therapy of urological diseases: standard versus alternative approaches
}

\author{
Kurt G. Naber ${ }^{1,6^{*}}$, Mikhail Kogan², Florian M. E. Wagenlehner ${ }^{3}$, Roswitha Siener ${ }^{4}$ and André Gessner ${ }^{5}$
}

\begin{abstract}
Until recently the generally accepted paradigm implied that urine of healthy people is sterile. In the meantime several studies have established also a microbiome in the bladder with many different species. Whether bacteria cause damage in the bladder depends not only on their virulence, but also on the inflammatory response of the host. Under certain circumstances asymptomatic bacteriuria can even protect from recurrent urinary tract infections (UTI). Some bacteria in the gut microbiome, such as Oxalobacter formigenes, are protective for calcium oxalate stone formation. The rapid rise of bacterial antibiotic resistance also among uropathogens due to wrong and often unreflected use of antibiotics has become a great concern. Instead of combating the pathogens, it appears to be more useful in many cases to treat the inflammatory host reaction - and to preserve the protective bacterial flora. Due to its antiphlogistic, spasmolytic and antinociceptive properties in a pilot study the herbal triad combination centaury, lovage, and rosmary leaves (CLR (Canephron ${ }^{\circledR}$ N (Bionorica SE, Neumarkt, Germany))) - showed very good results in the treatment of acute uncomplicated cystitis. In the meantime a phase 3 study with CLR in comparison with fosfomycin trometamol has started. Analysing microbiome profiles in mice showed that even a single dose of fosfomycin as well as daily application of nitrofurantoin resulted in massive microbiome shifts, whereas phytotherapy with CLR largely preserved the gut microbiota.
\end{abstract}

Keywords: Microbiome, Cystitis, Urinary tract infection, Phytotherapy

\section{The urinary microbiome in healthy individuals}

Based on enhanced urine culture techniques and sequencing of $16 \mathrm{~S}$ rDNA amplicons it was found that urine of healthy sexually active men and women is not sterile under normal conditions, which is in contrast to the generally accepted paradigm [1-3]. In the study of Kogan et al. [1] it was shown that in men and women, the group of facultative aerobic bacteria (FAB) is dominated by clusters of coagulase-negative staphylococci and Corynebacterium sp., and the group of nonclostridial anaerobic bacteria (NCAB) in women is dominated by clusters of Lactobacillus sp. and Peptococcus sp., and among men by Eubacterium sp. This knowledge of normal microbial communities in urine may alter the

\footnotetext{
* Correspondence: kurt@nabers.de

${ }^{1}$ Department of Urology, Technical University of Munich, Munich, Germany

${ }^{6}$ Karl-Bickleder-Str. 44c, 94315 Straubing, Germany

Full list of author information is available at the end of the article
}

standard diagnostic and therapeutic approaches to infectious and inflammatory diseases of the urogenital tract.
Review
Asymptomatic bacteriuria is normal
Since a high proportion of people are hosting bacteria in their urinary tracts, asymptomatic bacteriuria (ABU) is surprisingly common, which clearly should be differenti- ated from symptomatic urinary tract infection (UTI). Whether bacteria cause damage in the bladder depends not only on their virulence, but also on the inflamma- tory response of the host. The host and pathogens had developed a kind of peaceful coexistence in which both the virulence of the bacterial strain and the host re- sponse were downregulated. Colonization with specific strains of E. coli may even protect against symptom- atic UTI episodes: In a placebo-controlled study, pa- tients' bladder prone to recurrent UTI were instilled with the strain E. coli 83972 originally cultured from 
a patient with $\mathrm{ABU}$. The study not only demonstrated how effective this treatment is, it also suggested that similar E. coli strains may also prevent virulent and antibiotic-resistant bacteria from infecting the urinary tract [4].

\section{Pro or Con antibiotic treatment of asymptomatic bacteriuria?}

To test this theory, Cai et al. [5, 6] compared two different approaches to treating women prone to recurrent UTI and showing ABU between symptomatic episodes. Patients were split into two groups: $A B U$ of those in group A $(n=257)$ was not treated with antibiotics, while that of those in group B $(n=293)$ was. Group A showed a significant lower frequency of symptomatic UTI episodes than group B. Moreover $E$. coli strains from patients in group B showed significantly higher resistance rates to a range of antibiotics - including amoxicillinclavulanic acid, trimethoprim-sulfamethoxazole and ciprofloxacin. Thus, treating $\mathrm{ABU}$ with antibiotics might even be harmful [7].

Siener et al. [8] investigated the role of Oxalobacter formigenes, an oxalate-degrading bacterium that colonizes the intestinal tract, in calcium oxalate stone formation. The study revealed that patients with $\mathrm{O}$. formigenes in their gut microbiome had a significantly lower rate of stone recurrence. Furthermore, it showed that the absence of $\mathrm{O}$. formigenes in patients prone to kidney stones is likely due to the use of antibiotics, implying that antibiotic treatment may increase the risk of calcium oxalate stone formation.

\section{Antibiotic usage in acute urinary tract infections}

Every second woman has an acute UTI at least once during her life, $30 \%$ of women suffer from recurrent cystitis. Main pathogens are uropathogenic E. coli from the adjacent intestinal tract. Thus, the acute uncomplicated cystitis (AUC) in the outpatient area is the most frequent reason for prescribing an antibiotic and urology

Table 1 Antibiotic prescriptions for Outpatients in Germany in the Year 2014 [13]

\begin{tabular}{ll}
\hline Medical specialist & $\begin{array}{l}\mathrm{DDD}^{\mathrm{a}} \\
\text { per medical specialist }\end{array}$ \\
\hline ENT specialist & 5563 \\
Paediatrician & 5533 \\
Urologist & 5309 \\
Dermatologist & 5243 \\
General practitioner & 5003 \\
All medical specialists & 2186
\end{tabular}

adefined daily dose is one of the specialties with the highest outpatient antibiotic prescriptions (Table 1).

\section{How widespread is antibiotic resistance?}

The World Health Organization considers the rapid rise of bacterial antibiotic resistance among the three largest health problems worldwide. Wagenlehner [9] conducted a study in 2014 on antibiotic resistance in uropathogens like E. coli. It found that in Germany, $10-25 \%$ of E. coli was resistant to 3rd generation cephalosporin antibiotics, and 1$5 \%$ of Klebsiella pneumoniae showed even resistance to carbapenem antibiotics. The latter is particularly worrying, as carbapenem-resistant strains tend to be superbugs i.e. they are often resistant to all available antibiotics.

The study concluded that:

- Antibiotic resistance is widespread in patients with UTI

- Many strains have developed multi-drug resistance

- The more severe the infection, the higher the level of resistance

The wrong and often unreflected use of antibiotics may be responsible for that. These findings raise the question whether routine antibiotic therapy for AUC can still be considered useful - notleast because of the high spontaneous cure rates, the low complication risk and the increasing bacterial resistance development.

\section{Anti-inflammatory instead antimicrobial therapy}

The better understanding of the host-pathogen interaction leads to completely new therapeutic approaches. For example, in the case of uncomplicated UTI, broad-spectrum antibiotics can be avoided and antiinflammatory drugs can be used. Instead of combating the pathogens, it appears to be more useful in many cases to treat the inflammatory host reaction and to preserve the protective bacterial flora. This concept of "host tolerance" aims at a tolerance development of the host on the pathogen.

That antibiotics are not absolutely necessary to treat AUC in woman is demonstrated by a recent study comparing the symptom relief in AUC by an antiphlogistic drug, ibuprofen, with an antibiotic, fosfomycin. If the symptoms persisted or worsened, the patients of the ibuprofen arm could also switch to an antibiotic therapy. Nevertheless, two-thirds of women were able to be treated satisfactorily with anti-inflammatory therapy, only one in three women needed an antibiotic. On day seven, most patients were symptom-free. However, the symptoms in the fosfomycin group receded faster [10]. This study can be taken as proof of principles, but to search for better symptom-reducing drugs is still warranted. 


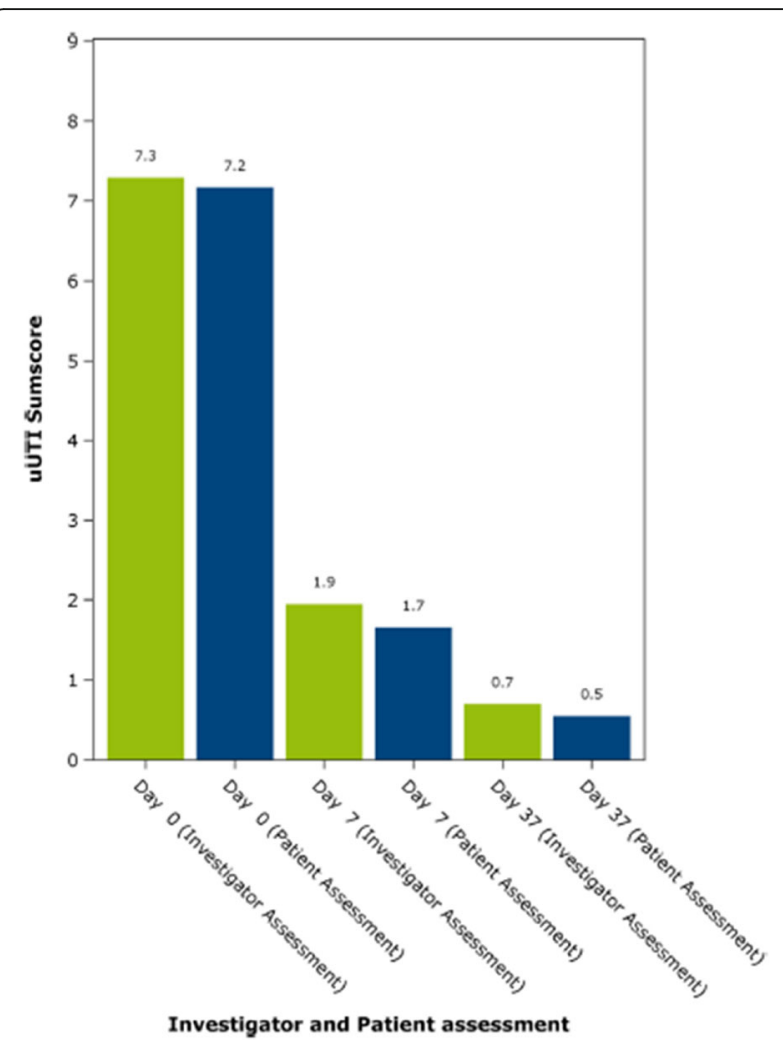

Fig. 1 Reduction of symptoms in female patients with acute uncomplicated cystitis (UUTI) treated with the phytodrug CLR for 7 days as assessed by patients and investigators [11]. The UUTI Sumscore includes the symptoms dysuria, frequency and urgency; UUTI Sumscore was assessed on Day 0, Day 7 and Day 37 (full analysis set, $N=125$ )

\section{Multitarget phytotherapy}

Multitarget phytotherapy may become relevant in the treatment of uncomplicated UTI. The herbal triad combination - centaury, lovage, and rosmary leaves $\left(C L R^{1}\right)$ has been used for decades for the supportive treatment of acute and recurrent UTI. CLR shows a broad spectrum of pharmacological activity. In addition to antiphlogistic effects, acute spasmolytic and antinociceptive properties also play an important role in acute therapy. Burning during micturition and spasms in the lower abdomen diminish. Additional anti-adhesive effects can prevent adherence and penetration of bacteria into the bladder mucosa. All these findings support the faster elimination of the pathogens and protect against recurrent infection.

\section{Promising pilot study}

In an open pilot study of 125 women with AUC were treated with the phytodrug CLR for 7 days [11]. Also in this study a switch to an antibiotic therapy was possible if the symptoms persisted or increased. Under this therapy, the severity of symptoms dropped sharply. Cystitisspecific symptoms such as dysuria, pollakisuria, and urinary urgency were only mild or decreased completely on day seven in $71 \%$ of the patients. On average, herbal therapy reduced the symptom score by $74 \%$ from 7.3 to 1.9 points (Fig. 1). Almost all women (98\%) did not need any antibiotic therapy. None of the successfully treated patients developed an early recurrence upto day 37 . The therapy was very well tolerated by the study participants; no adverse events were registered. Currently CLR is being tested in a Phase III study comparing the herbal triad combination with the antibiotic fosfomycin in women with AUC.
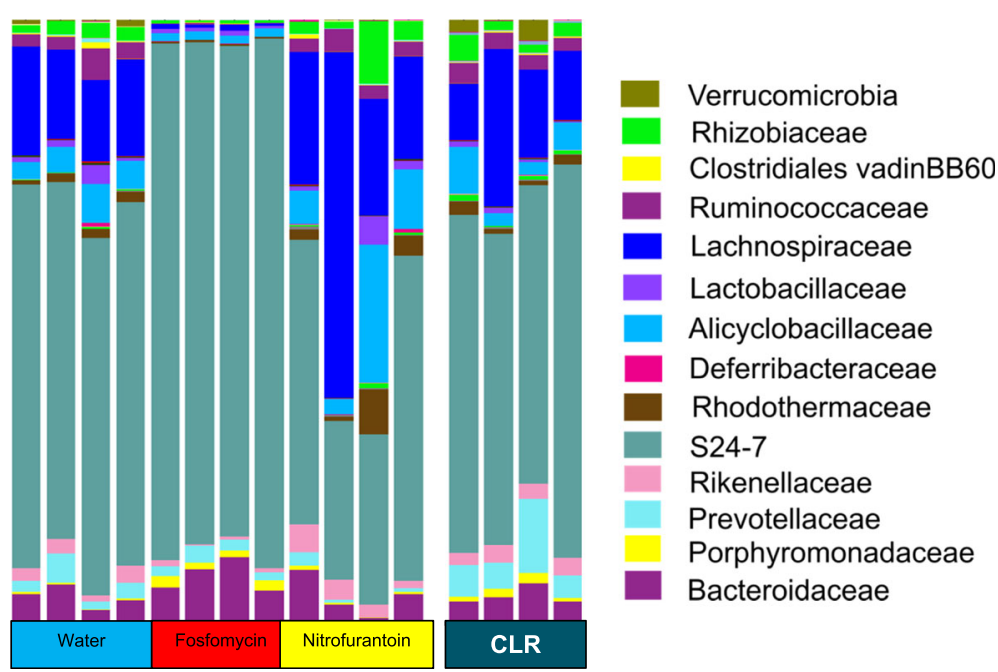

Fig. 2 Protection of microbiome with CLR in comparison to fosfomycin and nitrofurantoin after daily administration of CLR and antibiotics, respectively, by gavage in mice [12] 


\section{Microbiome preserving therapy}

A further advantage of the symptomatic therapy with the herbal triple combination is the low collateral damage compared to the antibiotic therapy. Many side effects of the antibiotics are caused by shifts in the body's bacterial colonization, e.g. gastrointestinal complaints, allergies or fungal infections in the vaginal area. Detailed knowledge about this has only been possible using modern DNA sequencing methods, since many bacteria cannot be detected with conventional cultivation methods at all.

Gessner et al. [12] investigated the intestinal microbiome of mice that received either a single dose of fosfomycin, 7 days of nitrofurantoin, water as a substancefree vehicle, or two different doses of the phytocombination. While the therapy with fosfomycin or nitrofurantoin showed massive microbiomic shifts, the intestinal bacterial flora remained largely unaffected by the phytocombination. The alpha-diversity, a measure of the biodiversity of the bacteria, was found in the faeces of the phytotherapeutically treated mice on a level with the faeces of the mice, which had only received water (Fig. 2). This was even the case when the plant combination was administered in a tenfold higher human equivalent dosage - a finding consistent with the very good tolerability of CLR in the pilot study. The faeces of the nitrofurantoin-treated mice, on the other hand, were clearly outside the normal range. This difference was even more pronounced in the mice who received single dose therapy with fosfomycin. Some bacterial families had even completely disappeared.

\section{Conclusion}

As shown by clinical studies the paradigm of antibiotic therapy for treatment of AUC is obviously changing. Instead of combating the pathogens, it may be more useful to treat the inflammatory host reaction to avoid collateral damage by the antibiotic treatment on the healthy microbiome. In this regard phythotherapeutic options could play a more important role and should be investigated further in prospective randomized clinical studies.

\section{Endnotes \\ ${ }^{1}$ Canephron $^{\circ} \mathrm{N}$ (Bionorica SE, Neumarkt, Germany)}

\section{Acknowledgement}

The REVIEW was sponsored by Bionorica SE, Neumarkt, Germany, using the material presented at a workshop held on 12th March 2016 as part of the 31st Annual European Association of Urology Congress in Munich, Germany

\section{Author's contributions}

KGN drafted the manuscript and all authors read and approved the manuscript.

\section{Competing interests}

The authors declare that they have no competing interest.

\section{Disclosure}

Kurt G. Naber: Investigator: Enteris Biopharma. Scientific Advisor (Review Panel or Advisory Committee): Bionorica, Enteris Biopharma, Helperby
Therapeutics, Leo Pharma, MerLion, MSD Sharp\&Dohme, OM Pharma, Paratek, Rosen Pharma, Zambon. Speaker's Bureau: Bionorica, DaiichiSankyo, Leo Pharma,OM Pharma, Rosen Pharma, Zambon.

Mikhail Kogan: Investigator: Pfizer, Astellas, Zambon, MSD, Shionogi, Ipsen, Scientifie Advisor: Besins, Ferron, Bionorica.

Florian Wagenlehner: Investigator: Enteris BioPharma. Scientific Advisor (Review Panel or Advisory Committee): Achaogen, AstraZeneca, Bionorica, Enteris BioPharma, Helperby Therapeutics, Janssen, Leo Pharma, MerLion, MSD, OM Pharma, Rosen Pharma, Shionogi.

Roswitha Siener: Scientific Advisor: Bionorica

André Gessner: Investigator: Bionorica, Roche, Speaker's Bureau: Bionorica, Falk Foundation, MSD Sharp\&Dohme, Roche, Pfizer.

\section{Author details}

${ }^{1}$ Department of Urology, Technical University of Munich, Munich, Germany. 2Department of Urology, Rostov State Medical University, Rostov, Russia. ${ }^{3}$ Department of Urology, Pediatric Urology and Andrology, Justus-Liebig University of Giessen, Giessen, Germany. ${ }^{4}$ University Stone Centre, Department of Urology and Pediatric Urology, University of Bonn, Bonn, Germany. ${ }^{5}$ Institute for Medical Microbiology and Hygiene, University of Regensburg, Regensburg, Germany. ${ }^{6}$ Karl-Bickleder-Str. 44c, 94315 Straubing, Germany.

Received: 1 February 2017 Accepted: 23 February 2017

Published online: 12 April 2017

References

1. Kogan MI, Naboka YL, Ibishev KS, Gudima IA, Naber KG. Human urine is not sterile - shift of paradigm. Urol Int. 2015;94(4):445-52.

2. Hilt EE, McKinley K, Pearce MM, et al. Urine is not sterile: use of enhanced urine culture techniques to detect resident bacterial flora in the adult female bladder. J Clin Microbiol. 2014;52(3):871-6.

3. Siddiqui H, Nederbragt AJ, Lagesen K, Jeansson SL, Jakobsen KS. Assessing diversity of the female urine microbiota by high throughput sequencing of 16S rDNA amplicons. BMC Microbiol. 2011;11:244.

4. Wullt B, Svanborg C. Deliberate establishment of asymptomatic bacteriuria a novel strategy to prevent recurrent UTI. Pathogens. 2016;5:52. doi:10.3390/ pathogens5030052.

5. Cai T, Mazzoli S, Mondaini N, Meacci F, Nesi G, D'Elia C, Malossini G, Boddi V, Bartoletti R. The role of asymptomatic bacteriuria in young women with recurrent urinary tract infections: to treat or not to treat? Clin Inf Dis. 2012;55(6):771-7.

6. Cai T, Nesi G, Mazzoli S, Meacci F, Lanzafame P, Caciagli P, Mereu L, Tateo S, Malossini G, Selli C, Bartoletti R. Asymptomatic bacteriuria treatment is associated with a higher prevalence of antibiotic resistant strains in women with urinary tract infections. Clin Infect Dis. 2015;61(11):1655-61.

7. Wagenlehner FME, Naber KG. Treatment of asymptomatic bacteriuria might be harmful. Clin Infect Dis. 2015;61(11):1662-3.

8. Siener R, Bangen U, Sidhu H, Hönow R, von Unruh G, Hesse A. The role of Oxalobacter formigenes colonization in calcium oxalate stone disease. Kidney Int. 2013;83:1144-9.

9. Wagenlehner FM, Bartoletti R, Cek M, Grabe M, Kahlmeter G, Pickard R, Bjerklund-Johansen TE. Antibiotic stewardship: a call for action by the urologic community. Eur Urol. 2013;64:358-60.

10. Gágyor I, Bleidorn J, Kochen MM, Schmiemann G, Wegscheider K, Hummers-Pradier E. Ibuprofen versus fosfomycin for uncomplicated urinary tract infection in women: randomised controlled trial. BMJ. 2015:351:h6544.

11. Ivanov D, Abramov-Sommariva D, Moritz K, Eskötter H, Kostinenko T, Martynyuk L, Kolesnik N, Naber KG. An open label, non-controlled, multicentre, interventional trial to investigate the safety and efficacy of Canephron ${ }^{\oplus} \mathrm{N}$ in the management of uncomplicated urinary tract infections (uUT/s). Clinical Phytoscience. 2015;1:7. doi:10.1186/s40816-015-0008-x.

12. Gessner A. The influence of urologic therapeutics on the microbiome in an experimental model" in "How the microbiome is influenced by the therapy of urological diseases: standard vs. alternative approaches". Munich: 31st Annual EAU Congress; 2016.

13. Schwabe U, Pfaffrath D, editors. Arzneiverordnungs-Report 2014. Springer 2014; cited according to Bundesamt für Verbraucherschutz und Lebensmittelsicherheit, Paul-Ehrlich-Gesellschaft für Chemotherapie e.V. GERMAP 2015 - Bericht über den Antibiotikaverbrauch und die Verbreitung von Antibiotikaresistenzen in der Human- und Veterinärmedizin in Deutschland. Rheinbach: Antiinfectives Intelligence; 2016. 